\title{
LIMITS ON EINSTEIN'S EQUIVALENCE PRINCIPLE FROM THE FIRST LOCALIZED FAST RADIO BURST FRB 150418
}

\author{
S. J. TingaY ${ }^{1}$ and D. L. Kaplan ${ }^{2}$ \\ ${ }^{1}$ International Centre for Radio Astronomy Research (ICRAR), Curtin University, Bentley, WA 6102, Australia; stingay@curtin.edu.au \\ ${ }^{2}$ Department of Physics, University of Wisconsin-Milwaukee, Milwaukee, WI 53201, USA; kaplan@uwm.edu \\ Received 2016 February 24; accepted 2016 March 9; published 2016 March 24
}

\begin{abstract}
Fast radio bursts (FRBs) have recently been used to place limits on Einstein's Equivalence Principle via observations of time delays between photons of different radio frequencies by Wei et al. These limits on differential post-Newtonian parameters $\left(\Delta \gamma<2.52 \times 10^{-8}\right)$ are the best yet achieved, but they still rely on uncertain assumptions, namely the relative contributions of dispersion and gravitational delays to the observed time delays and the distances to FRBs. Also, very recently, the first FRB host galaxy has likely been identified, providing the first redshift-based distance estimate to FRB 150418. Moreover, consistency between the $\Omega_{\mathrm{IGM}}$ estimate from FRB 150418 and $\Omega_{\mathrm{IGM}}$, expected from $\Lambda$ CDM models and WMAP observations, leads one to conclude that the observed time delay for FRB 150418 is highly dominated by dispersion, with any gravitational delays being small contributors. This points to even tighter limits on $\Delta \gamma$. In this paper, the technique of Wei et al. is applied to FRB 150418 to produce a limit of $\Delta \gamma<1-2 \times 10^{-9}$, approximately an order of magnitude better than previous limits and in line with expectations by Wei et al. for what could be achieved if the dispersive delay is separated from other effects. Future substantial improvements in such limits will depend on accurately determining the contribution of individual ionized components to the total observed time delays for FRBs.
\end{abstract}

Key words: cosmology: miscellaneous - gravitation - radio continuum: galaxies

\section{INTRODUCTION}

Fast radio bursts (FRBs), discovered by Lorimer et al. (2007) and reported by a range of teams since (Keane et al. 2012; Thornton et al. 2013; Burke-Spolaor \& Bannister 2014; Spitler et al. 2014; Champion et al. 2015; Masui et al. 2015; Petroff et al. 2015; Ravi et al. 2015; Keane et al. 2016), have been predicted to be fantastic cosmological and astrophysical probes (e.g., Macquart et al. 2015). They have additionally been shown by Wei et al. (2015) to be an excellent tool for constraining fundamental physics, namely Einstein's Equivalence Principle (EEP), if FRBs originate at cosmological distances. Wei et al. (2015) used limits on the gravitational time delay between photons of different radio frequencies generated by a FRB to infer upper limits on $\Delta \gamma=\gamma_{1}-\gamma_{2}$, where $\gamma_{n}$ is the post-Newtonian parameter describing how much space curvature is produced by a unit test mass (Misner et al. 1973) at the particular frequency of photon $n ; \gamma=1$ for general relativity.

Effectively, this treatment looks for whether or not photons of different energies "fall" at the same rate in a gravitational potential, ${ }^{3}$ in contrast to absolute limits on $\gamma-1$ that must be calibrated more carefully (Will 2014), with the best limits around $10^{-4}$ or $10^{-5}$ (Bertotti et al. 2003; Lambert \& Le Poncin-Lafitte 2011). Wei et al. (2015) infer their limits from their Equations (3) and (4):

$$
\begin{gathered}
\Delta t_{\mathrm{obs}}-\Delta t_{\mathrm{DM}}>\frac{\gamma_{1}-\gamma_{2}}{c^{3}} \int_{r_{o}}^{r_{e}} U(r) d r \\
\Delta \gamma=\gamma_{1}-\gamma_{2}<\left(\Delta t_{\mathrm{obs}}-\Delta t_{\mathrm{DM}}\right)\left[\frac{G M_{\mathrm{MW}}}{c^{3}} \ln \left(\frac{d}{b}\right)\right]^{-1},
\end{gathered}
$$

\footnotetext{
3 This test is similar to that performed on solar neutrinos (Gasperini 1988; Halprin \& Leung 1991; Gago et al. 2000), where the violation is a function of neutrino flavor rather than energy.
}

where $\Delta t_{\text {obs }}$ is the observed time delay between photons of different frequencies; $\Delta t_{\mathrm{DM}}$ is the time delay between photons of different frequencies due only to plasma dispersion, important at radio frequencies; $\gamma$ is as described above; $c$ is the speed of light; $r_{e}$ and $r_{o}$ are the positions of the emitter and the observer, respectively; $U(r)$ is the gravitational potential along the line of sight from the emitter to the observer; $G$ is the gravitational constant; $M_{\mathrm{MW}}$ is the mass of the Milky Way; $d$ is the distance between the emitter and the observer; and $b$ is the impact parameter of the emitted signal relative to the Milky Way center. Note that more accurate potential models can be used (e.g., Wu et al. 2016) but they change the results by at most $10 \%$. Wei et al. (2015) approximate the time delay between photons of two different frequencies due to the gravitational potential along the line of sight as $\Delta t_{\text {grav }}=\Delta t_{\text {obs }}-\Delta t_{\mathrm{DM}}$.

The fundamental observable for FRBs is $\Delta t_{\mathrm{obs}}$. For all detected FRBs the full value of $\Delta t_{\mathrm{obs}}$ has been equated with $\Delta t_{\mathrm{DM}}$ to infer a dispersion measure (DM) and therefore estimate a distance for each FRB, assuming knowledge of the ionized intergalactic medium (IGM) and the FRB's local environment. This indirect method of estimating distances has been necessary, as the single dish radio telescopes used to detect FRBs have lacked the angular resolution to identify any progenitor or host galaxy.

Wei et al. (2015) use such a line of reasoning (that $\Delta t_{\mathrm{obs}} \sim \Delta t_{\mathrm{DM}}$ ) to estimate the redshift, and therefore distance, to FRB 110220 (Thornton et al. 2013), but then require that the majority of $\Delta t_{\mathrm{obs}}$ is due to gravitational delay $\left(\Delta t_{\mathrm{obs}} \gg \Delta t_{\mathrm{DM}}\right)$ in order to produce a strict upper limit of $\Delta \gamma<2.52 \times 10^{-8}$ between the frequencies of $1.2 \mathrm{GHz}$ and $1.5 \mathrm{GHz}$. Wei et al. (2015) undertake a sensitivity analysis, given the uncertainties in the assumptions (distance to FRB and relative contributions of dispersion delay and gravitational delay to $\Delta t_{\mathrm{obs}}$ ), to show 

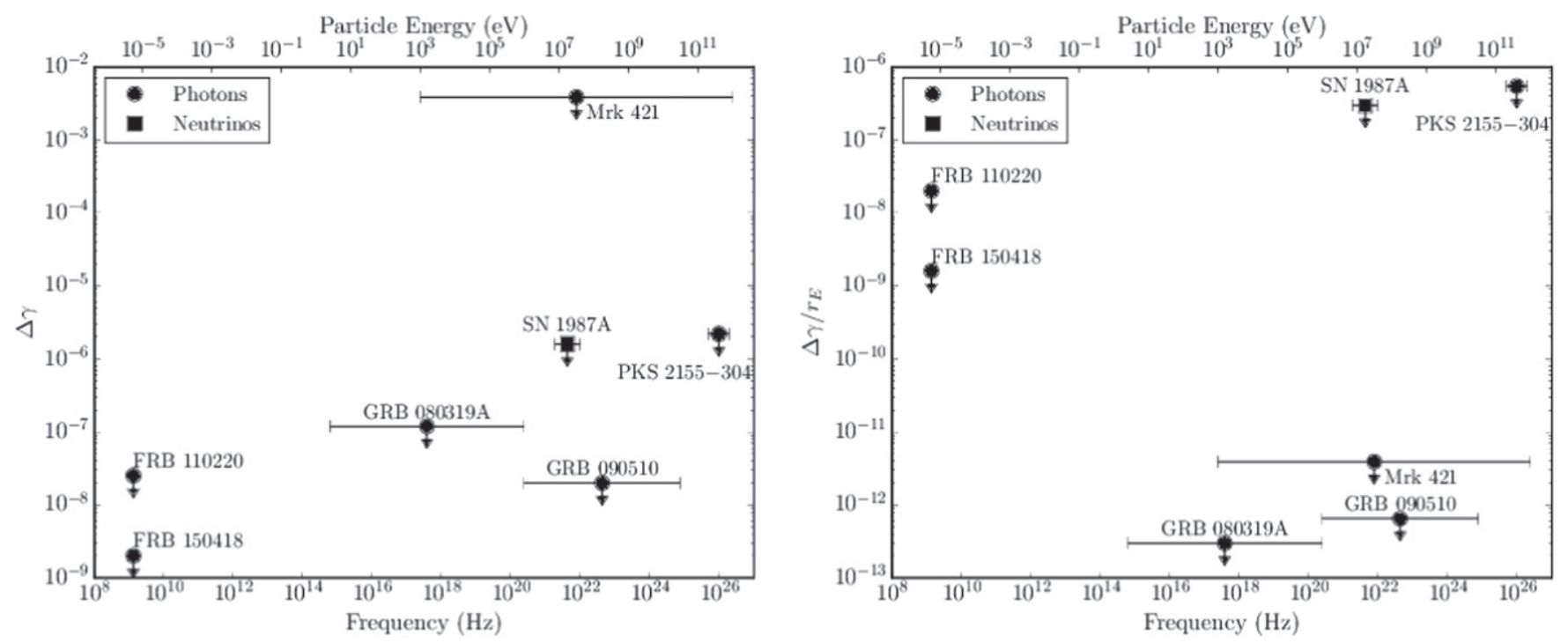

Figure 1. Left: differential limits on the post-Newtonian parameter $\gamma$ vs. particle energy or frequency, for two GRBs (Gao et al. 2015), SN 1987A (Longo 1988), FRB 110220 (Wei et al. 2015), the blazars Mrk 421 and PKS 2155-304 (Wei et al. 2016), and FRB 150418 (this work). The limit for SN 1987A is for neutrinos, while for the other sources the limit is for photons. In all cases the range of the error bars shows the range of particle energy used for the limit. Right: differential limits on $\gamma$ divided by the ratio of energies used $r_{E} \equiv E_{\mathrm{hi}} / E_{\mathrm{lo}}$ vs. particle energy or frequency.

that their limits are not particularly sensitive to the assumptions.

Wei et al. (2015) also produced limits for two proposed gamma-ray burst (GRB)/FRB associations. GRB 101011A and GRB 100704A were proposed to be associated with FRBlike radio transients by Bannister et al. (2012). Neither GRB has a redshift measurement, however, with distances estimated through the empirical Amati et al. (2002) relation. Moreover, further searches for FRB-like radio emission associated with GRBs, and a joint statistical analysis of a range of such experiments by Palaniswamy et al. (2014), show that the FRBlike signals reported by Bannister et al. (2012) are unlikely to be astrophysical. Thus, we do not further consider these as appropriate for deriving EEP limits. Conversely, the GRB-only constraints from Gao et al. (2015) are still valid.

Clearly, obtaining accurate distances to FRBs is one key advance required to utilize FRBs in the type of analysis proposed by Wei et al. (2015). Very recently the first accurate localization of an FRB led to the likely identification of an FRB host galaxy and the measurement of an FRB redshift (Keane et al. 2016). However, see Williams \& Berger (2016) for an alternate interpretation of the results presented in Keane et al. (2016).

\section{THE EEP CONSTRAINT FROM FRB 150418}

FRB 150418 was detected at the Parkes radio telescope on 2015 April 18 at 04:29:07.056 UT at a frequency of $1382 \mathrm{MHz}$, reported by Keane et al. (2016). Rapid multi-wavelength follow-up revealed a fading radio source at $\mathbf{J} 2000$ coordinates R.A. $=07^{\mathrm{h}} 16^{\mathrm{m}} 34^{\prime \prime} \cdot 55$, decl. $=-19^{\circ} 00^{\prime} 39^{\prime \prime} \cdot 9$, which Keane et al. (2016) argue is an afterglow associated with the FRB. At the position of the afterglow, imaging on the Subaru and Palomar 200 inch telescope and spectroscopy with Subaru and Keck reveal a $z=0.492 \pm 0.008$ galaxy, giving a luminosity distance of approximately $d=2.5 \mathrm{Gpc}$ (for an assumed cosmology of $H_{0}=69.6 \mathrm{~km} \mathrm{~s}^{-1} \mathrm{Mpc}^{-1}, \Omega_{m}=0.286$, and $\Omega_{\Lambda}=0.714$; Wright 2006).
From the celestial coordinates of FRB 150418, and taking the distance to the center of the Milky Way from Earth to be $8 \mathrm{kpc}$, the impact parameter is $b=6.4 \mathrm{kpc}$. The measured time delay, $\Delta t_{\mathrm{obs}}$, for FRB 150418 was approximately $0.8 \mathrm{~s}$, between frequencies of approximately 1.2 and $1.5 \mathrm{GHz}$. Using the same value for $M_{\mathrm{MW}}=6 \times 10^{11} M_{\odot}$ as used by Wei et al. (2015), and their assumption that $\Delta t_{\mathrm{obs}} \gg \Delta t_{\mathrm{DM}}$, the limit on $\Delta \gamma$ for FRB 150418 is $\Delta \gamma<2.2 \times 10^{-8}$, for photons of frequencies 1.2 and $1.5 \mathrm{GHz}$.

This limit is comparable to that inferred for FRB 110220 by Wei et al. (2015). However, after accounting for dispersion due to the host galaxy, the Milky Way, and the Milky Way halo, Keane et al. (2016) estimate that the remaining dispersion due to the IGM is $540 \pm 140 \mathrm{pc} \mathrm{cm}^{-3}$, which gives $\Omega_{\mathrm{IGM}}=0.049 \pm 0.013$ from the FRB 150418 data. This is compared to expectations from $\Lambda \mathrm{CDM}$ models and WMAP observations, which predict $\Omega_{\mathrm{IGM}}=0.041 \pm 0.002$ (Hinshaw et al. 2013). Both estimates agree to within the errors, leading to the reasonable conclusion that $\Delta t_{\mathrm{obs}}$ is dominated by dispersion and that the assumption adopted by Wei et al. (2015) $-\Delta t_{\mathrm{obs}} \gg \Delta t_{\mathrm{DM}}$-is not needed for FRB 150418 .

However, in the case of FRB 150418 it is still difficult to determine what fraction of $\Delta t_{\mathrm{obs}}$ could be attributable to gravitational delays. Within the errors, $\Delta t_{\text {obs }}$ for FRB 150418 is plausibly explained by dispersion in and around the Milky Way, in the host galaxy, and in the IGM. However, the errors in the various DM components are of the order of $20 \%$. For example, an error of $20 \%$ is quoted by Keane et al. (2016) on the largest non-IGM component of $\mathrm{DM}_{\mathrm{MW}}=189 \mathrm{pc} \mathrm{cm}^{-3}$, equating to $\sim 5 \%$ of the total DM. Also, a $100 \mathrm{pc} \mathrm{cm}^{-3}$ error is quoted on the IGM contribution to the DM, equating to $\sim 10 \%$ of the total DM. If errors in these quantities of $5 \%-10 \%$ magnitude masked gravitational delays, the limit on $\Delta \gamma$ above would be reduced by factors of 10-20, giving $\Delta \gamma<1.1 \times 10^{-9}$ (for a factor of 20). This limit is more than an order of magnitude lower than that obtained by Wei et al. (2015). We plot this limit along with other differential limits on $\gamma$ in Figure 1. 


\section{DISCUSSION}

With confirmation by Keane et al. (2016) that FRBs originate at cosmological distances, they are now serious contenders as cosmological probes and are of great interest for their intrinsic astrophysics. In addition to the fundamental advance provided by Keane et al. (2016), other recent results are starting to reveal information on their progenitors and environments (Masui et al. 2015). As suggested by Wei et al. (2015), future instruments such as the Square Kilometre Array (SKA) will be well-placed to open up new lines of investigation into astrophysics and cosmology using FRBs (Macquart et al. 2015).

Regarding the specific use of FRBs to place limits on Einstein's Equivalence Principle, the work of Wei et al. (2015) suggests great promise. With a distance now known for FRB 150418 , improved limits are now possible for this single FRB, likely approximately an order of magnitude better than that inferred by Wei et al. (2015). However, achieving further improvements in EEP limits using FRBs with the same technique will be difficult. The sensitivity of the limits to distance are not strong, as shown by Wei et al. (2015) since, for cosmological FRBs, $d$ will always be of order Gpc and $b$ will always be of order kpc, entering as $\ln (b / d)$ in the limit on $\Delta \gamma$. Incremental improvements for FRB 150418 may be possible through more realistic gravitational potentials for the Milky Way (e.g., Bovy 2015; Wu et al. 2016), the Local Group, and the host galaxy (once more mass estimates are available).

However, substantial improvements are possible via other sources of gravitational potential. Nusser (2016) argue that the potential from large-scale structure can lower the EEP limit by up to four orders of magnitude. This primarily comes from a statistical treatment of the fluctuations in gravitational potential estimated from the cosmic microwave background power spectrum (assuming linear structure growth). In a similar vein, Zhang (2016) estimate that EEP limits from FRBs could also be lowered by orders of magnitude if FRBs were identified behind galaxy clusters.

If the actual line of sight potential distribution is known for a burst-like FRB 150418 via optical or $\mathrm{H}_{\text {I }}$ galaxy redshift surveys (like Dawson et al. 2016 or Abdalla et al. 2015), a robust limit could be determined for individual objects. This would be in addition to the type of improvement in $\Delta \gamma$ discussed above.

A significant part of the value of the known distance for FRB 150418 is in the confirmation that dispersion dominates $\Delta t_{\mathrm{obs}}$. The challenge in placing future limits on EEP from FRBs will be in understanding the various dispersion components that contribute to this dominance: the Milky Way (and halo) contribution; the contribution from the FRB host galaxy; and the IGM contribution. These contributions are all uncertain at the $10 \%$ level. These uncertainties would need to be reduced to the $\sim 1 \%$ level to place confident limits on differential gravitational delays and thus attain $\Delta \gamma<10^{-10}$.

We can look to improve models of the Milky Way's ionized content. This will likely come from a combination of FRBs that are nearby (on the sky) to well-studied pulsars coupled with knowledge of the pulsar distances (Deller et al. 2011; Han et al. 2015). The SKA may be required to gather such observations, in particular pulsar distances via parallax measurements (Smits et al. 2011; Han et al. 2015). Separately, searches for pulsars/FRBs in Local Group galaxies (e.g., Bhat et al. 2011; Rubio-Herrera et al. 2013; Ravi et al. 2015) could constrain the dispersion measure of the halo. If more than one source can be detected in one of these galaxies (or even two cosmological FRBs in the same host galaxy), they would have common IGM and Milky Way (including halo) DM contributions but different host galaxy contributions. Such a scenario would provide some knowledge of the magnitude (and variability) of the host galaxy DM contribution.

Recently, Masui et al. (2015) detected Faraday rotation and scintillation for FRB 110523, providing evidence that this FRB occurred in a dense magnetized plasma environment (also see Kulkarni et al. 2015). Assuming that FRB 110523 originates in a host galaxy at cosmological distance, analyses such as performed by Masui et al. (2015) may, in the future, help provide some constraints on the DM contribution of the host galaxy. This may start to address the challenges noted above.

The limits presented here are differential limits, $\Delta \gamma$. Absent any particular form for EEP violation as a function of photon energy, we consider how our limits compare with those of Gao et al. (2015) and others by looking at $\Delta \gamma / r_{E}$, where $r_{E}=E_{\mathrm{hi}} / E_{\mathrm{lo}}$ is the ratio of high and low energies used in the limit. This is plotted in the right panel of Figure 1, and as can be seen the multi-decade spread in energies for GRBs and blazars (Wei et al. 2016) makes their limits in this metric better than FRB 150418 by more than three orders of magnitude. However, this could potentially be rectified by future improvements in FRB observing, as described above and below. Obtaining independent limits across a broad range of the electromagnetic (EM) spectrum will be useful if considering any model of EEP violation that has a frequency dependence, as well as studies of the plasma dispersion and constraining progenitor models through spectral indices (e.g., Karastergiou et al. 2015; Tingay et al. 2015). The recent direct detection of gravitational waves now opens up possibilities for similar tests using non-EM channels. For example, Ellis et al. (2016) calculate an upper limit to the difference in the speed of gravitational waves and gamma-rays from the possible coincidence of the GW150914 detection and a gamma-ray transient reported by Connaughton et al. (2016). Similarly, Wang et al. (2016) use the recent proposal that a gamma-ray outburst in PKS B1424-418 was associated with a PeV neutrino detection (Kadler et al. 2016) to place limits on EEP.

While most FRBs detected thus far have been between the frequencies of 1.2 and $1.5 \mathrm{GHz}$ at the Parkes radio telescope, FRB 110523 was detected between $700 \mathrm{MHz}$ and $900 \mathrm{MHz}$ with the Green Bank Telescope (Masui et al. 2015). Simultaneous detections at different telescopes may allow EEP limits over substantially larger frequency ranges, improving $\Delta \gamma / r_{E}$ significantly. In Keane et al. (2016) observations were done simultaneously with both Parkes at $1.4 \mathrm{GHz}$ and the Murchison Widefield Array (MWA) at $150 \mathrm{MHz}$, although the latter was a non-detection. Further FRB searches are active at low frequencies $(\sim 100 \mathrm{MHz})$ with the MWA (Tingay et al. 2015) and LOFAR (Coenen et al. 2014; Karastergiou et al. 2015) and at high frequencies $(10 \mathrm{~s}$ of $\mathrm{GHz})$ by the V-FASTR project at the VLBA (Trott et al. 2013). The larger frequency range also gives improved measurements of Faraday rotation $\left(\propto \nu^{-2}\right)$ and scattering $\left(\propto \nu^{-4}\right)$ that can help separate local and IGM contributions to time delay. Extending this to searches at optical and high-energy (X-ray, $\gamma$-ray) wavelengths could make this even more powerful, although there is not yet any evidence of simultaneous prompt emission from an FRB outside the radio band. 
Further opportunities for testing the EEP could come from the discovery of a multiply imaged FRB lensed by a galaxy (see Zheng et al. 2014), echoing the recent discovery of a multiply imaged supernova by Kelly et al. (2015b). ${ }^{4}$ This would require interferometric detection to achieve the needed spatial resolution, either with contemporary inteferometers (Law et al. 2015) or next-generation facilities like the SKA. In addition to the standard cosmological tests (Refsdal 1964), any deviations from the time delays expected purely from lensing could limit not just differential acceleration, $\Delta \gamma$, but the absolute acceleration $\gamma$. However, this relies on the precision of the lensing models, which, in the case of SN Refsdal is limited because of the massive galaxy, as well as a surrounding galaxy cluster. There could also be differences in dispersive delays through the cluster, but for typical intracluster medium densities of $\sim 10^{-3} \mathrm{~cm}^{-3}$ and lensing path delays of $\sim 10 \mathrm{~d}$, the difference in DM would be only $10^{-5} \mathrm{pc} \mathrm{cm}^{-3}$. Searches for FRBs toward massive galaxy clusters may be worthwhile even without the detection of of multiply imaged bursts, as the cluster would give an additional DM contribution of $\sim 10^{3} \mathrm{pc} \mathrm{cm}^{-3}$ (for a cluster size of $1 \mathrm{Mpc}$ ), which could be combined with measurements of the X-ray emission and the Sunyaev-Zeldovich (SZ) effect (Sunyaev \& Zeldovich 1980) to strongly constrain the hot gas in the cluster and aid in SZ cosmology. The polarization observed for FRBs may also be put to use in experiments utilizing a lensed FRB (Preuss 2003).

We thank an anonymous referee for helpful corrections. S.J.T. is a Western Australian Premier's Research Fellow. D.L.K. is supported by NSF grant AST-1412421.

\section{REFERENCES}

Abdalla, F. B., Bull, P., Camera, S., et al. 2015, Proceedings of Advancing Astrophysics with the Square Kilometre Array (AASKA14), 9-13 June, 2014 (Giardini Naxos, Italy), 17

Amati, L., Frontera, F., Tavani, M., et al. 2002, A\&A, 390, 81

Bannister, K. W., Murphy, T., Gaensler, B. M., \& Reynolds, J. E. 2012, ApJ, 757, 38

Bertotti, B., Iess, L., \& Tortora, P. 2003, Natur, 425, 374

Bhat, N. D. R., Cordes, J. M., Cox, P. J., et al. 2011, ApJ, 732, 14

Bovy, J. 2015, ApJS, 216, 29

Burke-Spolaor, S., \& Bannister, K. W. 2014, ApJ, 792, 19

Champion, D. J., Petroff, E., Kramer, M., et al. 2015, MNRAS, submitted, (arXiv:1511.07746)

Coenen, T., van Leeuwen, J., \& Hessels, J. W. T. O. 2014, A\&A, 570, A60

Connaughton, V., Burns, E., Goldstein, A., et al. 2016, arXiv:1602.03920

Dawson, K. S., Kneib, J.-P., Percival, W. J., et al. 2016, AJ, 151, 44

Deller, A. T., Brisken, W. F., Chatterjee, S., et al. 2011, in 20th Meeting of the European VLBI Group for Geodesy and Astronomy, ed. W. Alef,
S. Bernhart, \& A. Nothnagel (Bonn: Institut für Geodäsie und Geoinformation), 178

Ellis, J., Mavromatos, N. E., \& Nanopoulos, D. V. 2016, arXiv:1602.04764 Falcke, H., \& Rezzolla, L. 2014, A\&A, 562, A137

Gago, A. M., Nunokawa, H., \& Zukanovich Funchal, R. 2000, PhRvL, 84, 4035

Gao, H., Wu, X.-F., \& Mészáros, P. 2015, ApJ, 810, 121

Gasperini, M. 1988, PhRvD, 38, 2635

Halprin, A., \& Leung, C. N. 1991, PhRvL, 67, 1833

Han, J., van Straten, W., Lazio, J., et al. 2015, Advancing Astrophysics with the Square Kilometre Array (AASKA14), 9-13 June, 2014 (Giardini Naxos, Italy), 41

Hinshaw, G., Larson, D., Komatsu, E., et al. 2013, ApJS, 208, 19

Kadler, M., Krauß, F., Mannheim, K., et al. 2016, NatPh, in press (arXiv:1602.02012)

Karastergiou, A., Chennamangalam, J., Armour, W., et al. 2015, MNRAS, 452,1254

Keane, E. F., Johnston, S., Bhandari, S., et al. 2016, Natur, 530, 453

Keane, E. F., Stappers, B. W., Kramer, M., \& Lyne, A. G. 2012, MNRAS, 425, L71

Kelly, P. L., Rodney, S. A., Brammer, G., et al. 2015a, ATel, 8402

Kelly, P. L., Rodney, S. A., Treu, T., et al. 2015b, Sci, 347, 1123

Kulkarni, S. R., Ofek, E. O., \& Neill, J. D. 2015, arXiv:1511.09137

Lambert, S. B., \& Le Poncin-Lafitte, C. 2011, A\&A, 529, A70

Law, C. J., Bower, G. C., Burke-Spolaor, S., et al. 2015, ApJ, 807, 16

Longo, M. J. 1988, PhRvL, 60, 173

Lorimer, D. R., Bailes, M., McLaughlin, M. A., Narkevic, D. J., \& Crawford, F. 2007, Sci, 318, 777

Macquart, J. P., Keane, E., Grainge, K., et al. 2015, in Advancing Astrophysics with the Square Kilometre Array (AASKA14), 9-13 June, 2014 (Giardini Naxos, Italy), 55

Masui, K., Lin, H.-H., Sievers, J., et al. 2015, Natur, 528, 523

Misner, C. W., Thorne, K. S., \& Wheeler, J. A. 1973, Gravitation (San Francisco: Freeman)

Nusser, A. 2016, ApJL, in press (arXiv:1601.03636)

Palaniswamy, D., Wayth, R. B., Trott, C. M., et al. 2014, ApJ, 790, 63

Petroff, E., Bailes, M., Barr, E. D., et al. 2015, MNRAS, 447, 246

Preuss, O. 2003, PhD thesis, Universität Bielefeld

Ravi, V., Shannon, R. M., \& Jameson, A. 2015, ApJL, 799, L5

Refsdal, S. 1964, MNRAS, 128, 307

Rubio-Herrera, E., Stappers, B. W., Hessels, J. W. T., \& Braun, R. 2013 , MNRAS, 428, 2857

Smits, R., Tingay, S. J., Wex, N., Kramer, M., \& Stappers, B. 2011, A\&A, 528, A108

Spitler, L. G., Cordes, J. M., Hessels, J. W. T., et al. 2014, ApJ, 790, 101

Sunyaev, R. A., \& Zeldovich, I. B. 1980, ARA\&A, 18, 537

Thornton, D., Stappers, B., Bailes, M., et al. 2013, Sci, 341, 53

Tingay, S. J., Trott, C. M., Wayth, R. B., et al. 2015, AJ, 150, 199

Trott, C. M., Tingay, S. J., Wayth, R. B., et al. 2013, ApJ, 767, 4

Wang, Z.-Y., Liu, R.-Y., \& Wang, X.-Y. 2016, arXiv:1602.06805

Wei, J.-J., Gao, H., Wu, X.-F., \& Mészáros, P. 2015, PhRvL, 115, 261101

Wei, J.-J., Wang, J.-S., Gao, H., \& Wu, X.-F. 2016, ApJL, 818, L2

Will, C. M. 2014, LRR, 17, 4

Williams, P. K. G., \& Berger, E. 2016, arXiv:1602.08434

Wright, E. L. 2006, PASP, 118, 1711

Wu, X.-F., Gao, H., Wei, J.-J., et al. 2016, arXiv:1602.01566

Zhang, B. 2014, ApJL, 780, L21

Zhang, S.-N. 2016, A\&A, submitted, (arXiv:1601.04558)

Zheng, Z., Ofek, E. O., Kulkarni, S. R., Neill, J. D., \& Juric, M. 2014, ApJ, 797,71

\footnotetext{
4 In the future, one could also conduct FRB search observations contemporaneously with searches for the late-appearing lensed component of multiply imaged supernovae (Kelly et al. 2015a), to constrain progenitor models (e.g., Falcke \& Rezzolla 2014; Zhang 2014).
} 